\title{
On Lyapunov Sampling for Event-driven Controllers
}

\author{
Manel Velasco, Pau Martí and Enrico Bini
}

\begin{abstract}
This paper investigates an event condition for event-driven controllers based on Lyapunov functions. Considering that constant values of a Lyapunov function define contour curves that form closed regions around the equilibrium point, in this paper we present a sampling mechanism that enforces job executions (sampling, control algorithm computation and actuation) each time the system trajectory reaches a given contour curve. By construction, the sequence of generated samples is stable in the discrete Lyapunov sense. However, in order to ensure that the system trajectory will tend to zero as time tends to infinity, it must be ensured that the sequence of samples is infinite. We provide conditions to ensure this property. The approach is illustrated by simulated examples.
\end{abstract}

\section{INTRODUCTION}

Computing and communication capabilities are being embedded in all types of objects and structures in the physical environment, creating the so-called cyber-physical systems (CPSs) [1]. For these systems, sensing and control of physical entities, that is, feedback control loops, require new paradigms like breaking the notion that sample times have to be equidistant, constant, and synchronized [2]. This may lead a more efficient use of networks and processors while ensuring or maximizing control performance, as well as a better adaptation of networked applications to different and dynamic time scales.

In this line, recent research has provided diverse theoretical results suggesting that for several control loops sharing limited computing resources, a key design aspect is to efficiently select the controllers' sampling periods, e.g. [3], [4], [5], [6], [7], [8], [9], [10], [11], [12], [13], [14]. These results indicate that controllers' execution rates must be different from those provided by the standard periodic sampling approach, either tackling the problem of sampling period selection using feedback scheduling techniques or event-driven control techniques.

Within the context of event-driven control systems, in this paper we investigate a new sampling scheme based on Lyapunov functions. A set of contour curves of the Lyapunov function define a discretization in a energy space domain, and the system trajectory can move between them without requiring control actions. Control jobs are only activated each time the trajectory intersects a contour curve.

Recently, event-driven control systems have been receiving increased attention. Controller jobs are triggered following

This work was partially supported by C3DE Spanish CICYT DPI200761527 and by ArtistDesign NoE IST-2008-214373.

M. Velasco and P. Martí are with the Automatic Control Department, Technical University of Catalonia, Pau Gargallo 5, 08028 Barcelona, Spain \{manel.velasco, pau.marti\}@upc.edu

E. Bini with the ReTiS Lab, Scuola Superiore Sant'Anna, Via G. Moruzzi 1, 56124, Pisa, Italy e.bini@sssup.it

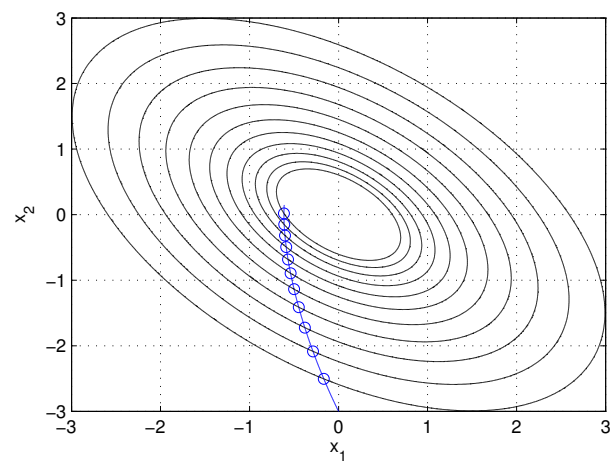

Fig. 1: Lyapunov sampling mechanism

different mechanisms, such as diverse forms of level-crossing mechanisms [5], [6], [7], [10], or state or self-triggered mechanisms [3], [4]. However, none of them has presented a sampling mechanism based on Lyapunov functions as we do, although some closely related work can be found in [15].

\section{A. Key points and problems to be solved}

Figure 1 illustrates the proposed sampling mechanism. In the figure, the Lyapunov function is represented in the $\left(x_{1}, x_{2}\right)$ plane by ellipses (contour curves) of constant energy. Jobs executions, represented by circles, are only enforced each time the system trajectory (solid line) intersects a contour curve from outside to inside, i.e., decreasing the system energy. Therefore, by construction, the generated samples are stable in the discrete Lyapunov sense. We call this triggering mechanism "Lyapunov sampling".

Although the generated sequence of samples is stable in the Lyapunov sense, i.e. decreasing energy at each sample, the stability of the continuous dynamics are not guaranteed. That is, from the sequence of samples, it cannot be ensured that the system trajectory will tend to zero as time progresses because the sequence of samples can be finite, as we will further illustrate in the paper.

Note also that ensuring an infinite sequence of samples implies that all sampling intervals are bounded. This facilitates the assessment of the controller computational demand, thus permitting the feasibility analysis of a set of concurrent event-driven controllers [16]. In addition, if sampling intervals can be predicted, more efficient techniques can be applied to the design of the controller.

Problem 1: The first problem to be solved is to ensure that the Lyapunov sampling mechanism produces an infinite sequence of samples. 
The application of the Lyapunov sampling mechanism requires dedicated hardware logics to check the system trajectory and check whether the control job must be activated. However, if the next activation time can be computed at each job execution, the dedicated hardware is no longer required. Jobs will follow a self-triggered model [17] because each job will determine the release of its next job.

Problem 2: The second problem to be solved is to find a technique that allows a self-triggered scheme for the Lyapunov sampling mechanism.

\section{B. Paper contribution and structure}

The contribution of this paper is to present a novel execution rule for event-driven control systems intended to drive the system to a neighborhood of the equilibrium.

The rest of this paper is structured as follows. Section II presents the preliminaries and illustrates some of the concepts outlined earlier. Section III presents the Lyapunov sampling mechanism that ensures stability of the system trajectory, thus solving Problem 1. The feasibility of computing a key parameter of the introduced sampling mechanism is discussed in Section IV. Section V addresses the selftriggered approach for the Lyapunov sampling, addressing Problem 2. And Section VI concludes the paper.

\section{PRELIMINARIES}

\section{A. Event-driven control system model}

We consider the continuous control system

$$
\dot{x}(t)=f(x(t), u(t))
$$

where $x \in \mathbb{R}^{n}$ denotes the state and $u \in \mathbb{R}^{m}$ the input.

Let

$$
\forall t \in\left[t_{i}, t_{i+1}\right) \quad u(t)=k\left(x\left(t_{i}\right)\right)=k\left(x_{i}\right)
$$

be the control updates given by a feedback controller $k$ : $\mathbb{R}^{n} \rightarrow \mathbb{R}^{m}$ using only samples of the state at discrete instants

$$
t_{0}, t_{1}, \ldots, t_{i}, \ldots
$$

With (2), the closed loop system becomes

$$
\dot{x}(t)=f\left(x(t), k\left(x_{i}\right)\right) .
$$

\section{B. Lyapunov sampling}

Definition 1: Let $V: \mathbb{R}^{n} \rightarrow \mathbb{R}$ be a (local or global) Lyapunov function in the classical sense, i.e., continuous and positive definite. We call $\mathcal{L}_{s}$ the set of sequences of samples obtained from evaluating system (4) in the sampling instants (3) given by the Lyapunov sampling triggering mechanism

$$
V\left(x\left(t_{i+1}\right)\right)=\eta V\left(x\left(t_{i}\right)\right) \quad, \quad \eta \in \mathbb{R}^{+} .
$$

From Eq. (5), we notice that for small values of $\eta$ we expect large sampling periods. In fact, small values of $\eta$ mean that we set the next sampling instant when the Lyapunov function has decreased more significantly with respect to the current value.

For a given initial condition $x_{0}, \mathcal{L}_{s}\left(x_{0}\right)$ represents a particular sequence generated as indicated previously.

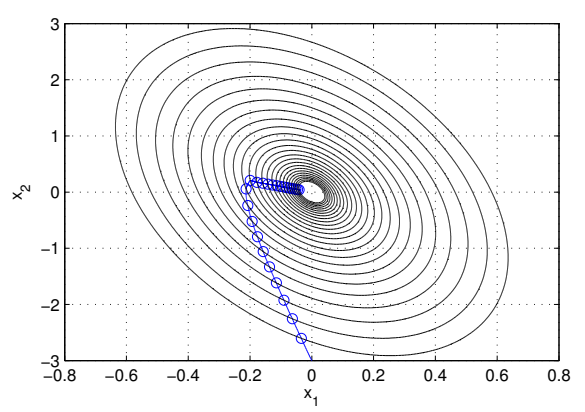

(a) Sample sequence and continuous dynamics

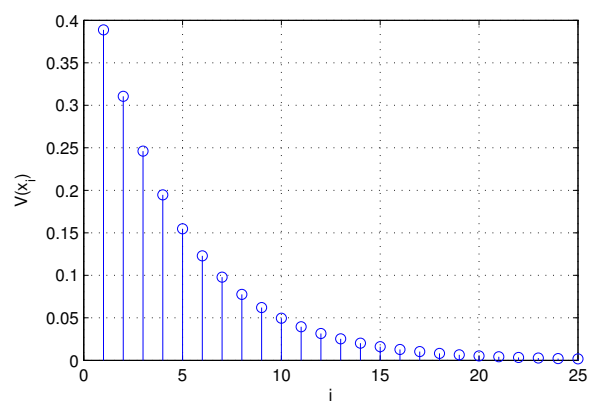

(b) Energy at the sampling instants

Fig. 2: Stable sequence and stable dynamics

Lemma 1: By construction, it follows that $\mathcal{S L}_{s} \in \mathcal{L}_{s}$ is the subset of sequences that are stable in the discrete Lyapunov sense if $\eta$ in the Lyapunov sampling triggering mechanism (5) is restricted to

$$
0<\eta<1 \text {. }
$$

Note that $V$ is not required to guarantee stability in the continuous Lyapunov sense for the closed loop continuous system (4). In addition, no restriction is set on the controller law $k(\cdot)$.

Lemma 1 guarantees stable sampling sequences. However, nothing is ensured about the stability of the continuous-time dynamics (4) when $x_{i} \in \mathcal{S} \mathcal{L}_{s}\left(x_{0}\right)$.

\section{Illustrative examples}

In this section we present two examples that illustrate two opposite behaviors for the double integrator continuous time dynamics when the samples are stable in the discrete Lyapunov sense.

Example 1: Throughout the paper, we will illustrate the different results using a simple double integrator system

$$
\dot{x}=A x+B u
$$

where

$$
A=\left[\begin{array}{ll}
0 & 1 \\
0 & 0
\end{array}\right], B=\left[\begin{array}{l}
0 \\
1
\end{array}\right] .
$$

The Lyapunov function is

$$
V(x)=x^{T} P x
$$




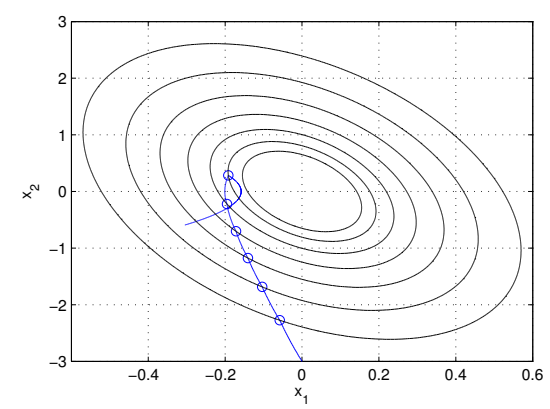

(a) Sample sequence and continuous dynamics

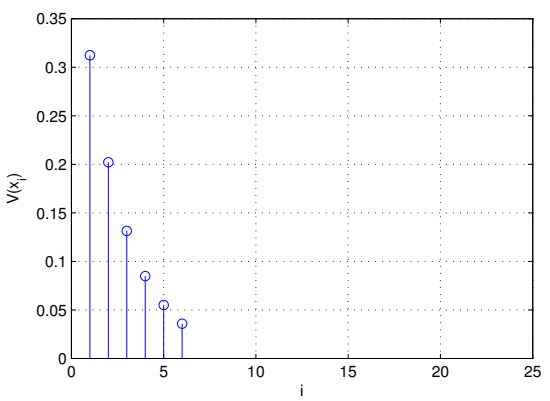

(b) Energy at the sampling instants

Fig. 3: Stable sequence and unstable dynamics

where

$$
P=\left[\begin{array}{cc}
1.1455 & 0.1 \\
0.1 & 0.0545
\end{array}\right] .
$$

The initial condition is

$$
x_{0}=\left[\begin{array}{c}
0 \\
-3
\end{array}\right] .
$$

And control updates are given by the linear state feedback gain

$$
L=[-10-11] \text {. }
$$

Example 2: Consider the simulation setting of Example 1 with the Lyapunov sampling mechanism (5) with $\eta=0.8$, that is, samples are taken when $V\left(x\left(t_{i+1}\right)\right)=0.8 V\left(x\left(t_{i}\right)\right)$ holds. Figure 2 (a) plots the closed loop continuous-time trajectory and the sequence of generated samples (circles) on top of the contour curves. And Figure (b) plots the energy $V\left(x_{i}\right)$ at each sample. As it can be observed, the sequence of samples is stable and the trajectory is stable. Due to image resolution, only 25 samples are shown. However, as the time progresses, more samples are taken, driving the trajectory toward zero.

Example 3: Consider the simulation setting of Example 2 with $\eta=0.65$. Figure 3 (a) and (b) plot the same information as before. As it can be observed, although the sequence of samples is stable in the discrete Lyapunov sense, the continuous-time trajectory is unstable. Note that the sequence of samples is finite, and from the sixth sample, no further samples are taken.

These examples illustrate the problems outlined previously in Section I-A. Next section presents the sampling mechanism that generates an infinite sequences of samples, thus ensuring stability of the continuous-time dynamics, called "stable Lyapunov sampling".

\section{STABLE LYAPUNOV SAMPLING}

Looking at Figure 3 (a), from the last sample, we have to determine the energy decrease produced by the system trajectory up to the point it starts gaining energy again. Placing a contour curve passing for that point would have ensured a new sample. Therefore, for any initial condition, i.e. current state, we have to determine the bound on energy decrease produced by the system trajectory that still guarantees the occurrence of the next sample.

And intuitively, this strongly relates to $\eta$. In fact, in this section we derive further restrictions for $\eta$ in (5) in such a way that the generated sequence of samples is infinite.

Definition 2: Let $x\left(t, x_{0}\right)$ be the solution of (4), when $u(t)=k\left(x_{0}\right)$, where $x_{0}$ is a given initial condition. Let the function $V^{*}: \mathbb{R}^{n} \rightarrow \mathbb{R}$

$$
V^{*}\left(x_{0}\right)=\min _{t} V\left(x\left(t, x_{0}\right)\right) \quad \forall t \geq 0
$$

denote the minimum achieved energy without changing the control signal for any initial condition. And let $t^{*}: \mathbb{R}^{n} \rightarrow \mathbb{R}$

$$
t^{*}\left(x_{0}\right)=\min \left(\arg \min _{t} V\left(x\left(t, x_{0}\right)\right)\right)
$$

be the time at which the minimum energy is achieved.

We assume that the minimum in (7) exists.

Taking into account the initial energy $V\left(x_{0}\right)$ and the minimum achieved energy $V^{*}\left(x_{0}\right)$ given by (7) for any initial condition, we are interested in finding the minimum distance between them, that is,

$$
\min _{x_{0}} V\left(x_{0}\right)-V^{*}\left(x_{0}\right),
$$

which is always a positive quantity by construction. Normalizing (9), we obtain

$$
\min _{x_{0}} 1-\frac{V^{*}\left(x_{0}\right)}{V\left(x_{0}\right)} .
$$

The previous minimum will occur when $\frac{V^{*}\left(x_{0}\right)}{V\left(x_{0}\right)}$ is maximum. Let the function $\hat{\eta}: \mathbb{R}^{n} \rightarrow \mathbb{R}$ be

$$
\hat{\eta}\left(x_{0}\right)=\frac{V^{*}\left(x_{0}\right)}{V\left(x_{0}\right)} .
$$

Definition 3: Let

$$
\eta^{*}=\max _{x_{0}} \hat{\eta}\left(x_{0}\right) .
$$

be the energy gain factor that minimizes (9). Note that by construction, $0 \leq \eta^{*} \leq 1$.

Example 4: Figure 4 illustrates the concept given by definition 2 in the context of example 2. In Figure 4 we plot $\mathrm{V}(\cdot)$ for two initial conditions, namely $x_{i}^{1}$ and $x_{i}^{2}$ (with solid and dashed lines respectively). In this case, both initial conditions belong to the same contour curve $V(x)=0.015$. As it can be seen, for $x_{i}^{1}$ the minimum energy is $V^{*}\left(x_{i}^{1}\right)=0.005$ and the time it occurs is $t^{*}\left(x_{i}^{1}\right)=0.117 \mathrm{~s}$. For $x_{i}^{2}, V^{*}\left(x_{i}^{2}\right)=0.003$ and $t^{*}\left(x_{i}^{2}\right)=0.103 \mathrm{~s}$. Looking at definition 3 , and by only considering these two initial conditions, observe that 


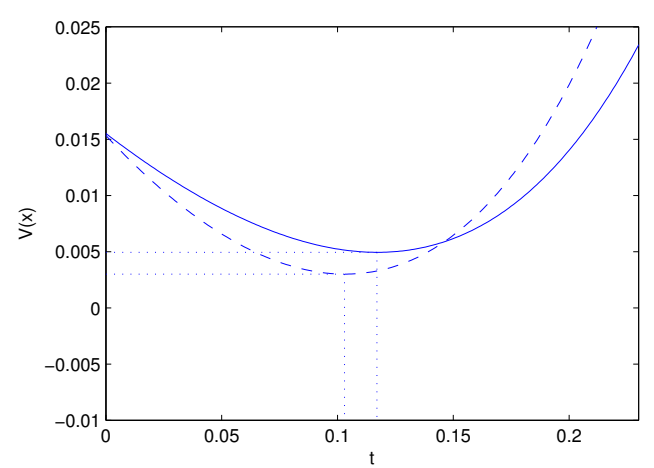

Fig. 4: Looking for the minimum achieved energy

$\hat{\eta}\left(x_{i}^{1}\right)=0.33$ and $\hat{\eta}\left(x_{i}^{2}\right)=0.2$. Therefore, with this example, $\eta^{*}=0.33$. Note that 0.33 generates a denser discretization of contour curves than 0.2 . However, the computation of $\eta^{*}$ must be for any initial state.

Theorem 1: For any $x_{0}$, if $\eta^{*}<\eta<1$ then $\mathcal{S} \mathcal{L}_{s}\left(x_{0}\right)$ is an infinite sequence.

The theorem presents the stable Lyapunov sampling mechanism, which is based on restricting $\eta$ in the Lyapunov sampling condition (5) in such a way that the space discretization given by the set of contour curves ensures infinite samples.

Proof: To prove that $\mathcal{S} \mathcal{L}_{s}\left(x_{0}\right)$ is an infinite sequence, we will prove that for any sample $x_{i} \in \mathcal{S} \mathcal{L}_{s}\left(x_{0}\right)$, the next sample $x_{i+1} \in \mathcal{S} \mathcal{L}_{s}\left(x_{0}\right)$ exists.

The event is activated according (5). From (5) let the continuous function $G: \mathbb{R} \rightarrow \mathbb{R}$ be

$$
G(t)=V(x(t))-\eta V\left(x_{i}\right) .
$$

We are interested in the roots of $G$, i.e., when (5) holds.

- For $t=t_{i}$, i.e., at the current state, $G\left(t_{i}\right)>0$. Note that

$$
\begin{aligned}
G\left(t_{i}\right) & =V\left(x\left(t_{i}\right)\right)-\eta V\left(x_{i}\right) \\
& =V\left(x_{i}\right)-\eta V\left(x_{i}\right) \\
& =(1-\eta) V\left(x_{i}\right) .
\end{aligned}
$$

Since $V\left(x_{i}\right)>0$ and $1-\eta>0, G\left(t_{i}\right)>0$.

- For $t=t^{*}\left(x_{i}\right)$, i.e., at the time the minimum energy is achieved, $G\left(t^{*}\right)<0$. Note that

$$
\begin{aligned}
G\left(t^{*}\left(x_{i}\right)\right) & =V\left(x\left(t^{*}\left(x_{i}\right)\right)\right)-\eta V\left(x_{i}\right) \\
& =V^{*}\left(x_{i}\right)-\eta V\left(x_{i}\right) \\
& <V^{*}\left(x_{i}\right)-\eta^{*} V\left(x_{i}\right) \\
& =V^{*}\left(x_{i}\right)-\max _{x_{0}} \frac{V^{*}\left(x_{0}\right)}{V\left(x_{0}\right)} V\left(x_{i}\right) \\
& \leq V^{*}\left(x_{i}\right)-\max _{x_{i}} \frac{V^{*}\left(x_{i}\right)}{V\left(x_{i}\right)} V\left(x_{i}\right) \\
& \leq V^{*}\left(x_{i}\right)-\frac{V^{*}\left(x_{i}\right)}{V\left(x_{i}\right)} V\left(x_{i}\right) \\
& =V^{*}\left(x_{i}\right)-V^{*}\left(x_{i}\right) \\
& =0 .
\end{aligned}
$$

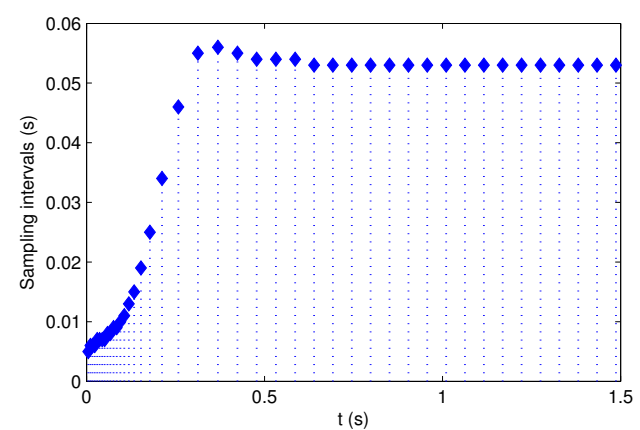

Fig. 5: Sampling intervals

Due to Bolzano theorem, if $G\left(t_{i}\right)>0$ and $G\left(t^{*}\right)<0$ and taking into account that $G$ is continuous in $\left[t_{i}, t^{*}\right]$, it $\exists t \in$ $\left(t_{i}, t^{*}\right)$, namely $t_{i+1}$ such that $G\left(t_{i+1}\right)=0$, i.e., the event is activated and the next sample $x_{i+1}$ is taken.

Corollary 1: If the sequence of samples is infinite, a maximum sampling interval exists and it is bounded by $t^{*}-t_{i}$.

Example 5: From the Example 2, we can find numerically that $\eta^{*}=0.7818$, which is given by any initial condition that points into the direction $\left[\begin{array}{ll}0.5069 & -0.8620\end{array}\right]$. Remind that for Figure 2, with stable dynamics, $\eta^{*}<\eta=0.8$, and for Figure 3, with unstable dynamics, $\eta^{*}>\eta=0.65$, which corroborates the restrictions in $\eta$ imposed in the theorem. The bound on the maximum sampling interval is $t^{*}-t_{i}=2.9216$. For illustrative purposes, Figure 5 shows the beginning of the sequence of sampling intervals for the Example 2. The $x$-axis is simulation time (note that only $1.5 \mathrm{~s}$ are displayed), and the $y$-axis is the sampling interval in seconds. Each sampling interval is represented by a vertical line, separated by its value. Hence it can be noticed that denser controller job activations occur when the heights are shorter. Surprisingly, sampling periods evolve from short values to high values, settling to a given value of $0.052 \mathrm{~s}$, being all of them shorter than the derived bound.

\section{COMPUTATION OF $\eta^{*}$ FOR LINEAR SYSTEMS}

In example 5 we indicated that $\eta^{*}$ was found numerically. It is important to note that computing $\eta^{*}$ for non-linear systems is not trivial because it is a non-convex problem. For linear systems, although being also non-convex, some simplifications can be performed. This permits to derive a feasible numerical algorithm for finding $\eta^{*}$.

Hence, restricting our interest to linear systems and linear controllers, and aligned with some results presented in [18], the following two scaling lemmas hold.

Lemma 2: The system solution with constant input scales with a given initial condition, i.e.

$$
x\left(t, c x_{0}\right)=c x\left(t, x_{0}\right) \quad, \quad c \in \mathbb{R} .
$$

Proof: Given the system solution

$$
x\left(t, x_{0}\right)=e^{A t} x_{0}+\int_{0}^{t} e^{A s} d s L x_{0},
$$




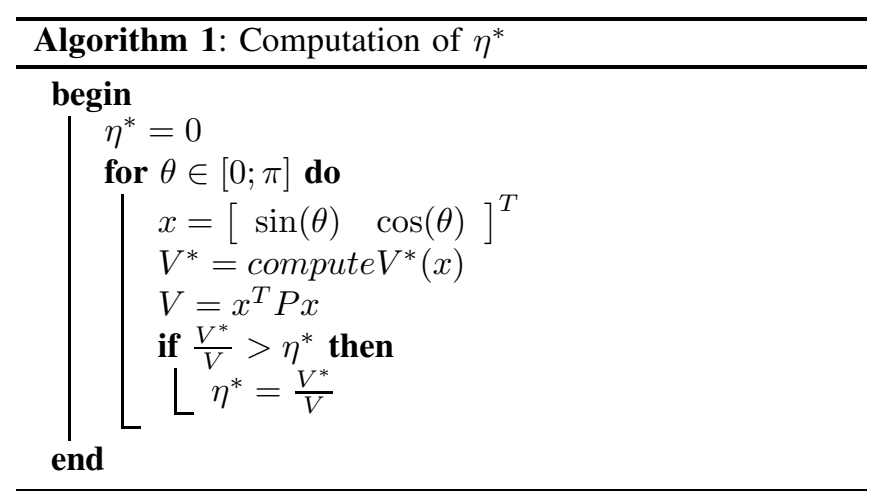

it can be easily seen that

$$
\begin{aligned}
x\left(t, c x_{0}\right) & =e^{A t} c x_{0}+\int_{0}^{t} e^{A s} d s L c x_{0} \\
& =c\left(e^{A t} x_{0}+\int_{0}^{t} e^{A s} d s L x_{0}\right) \\
& =c x\left(t, x_{0}\right) .
\end{aligned}
$$

Lemma 3: The energy in terms of the Lyapunov function scales with a given system solution, i.e.

$$
V\left(x\left(t, c x_{0}\right)\right)=c^{2} V\left(x\left(t, x_{0}\right)\right) \quad, \quad c \in \mathbb{R} .
$$

Proof: Given the energy

$$
V\left(x\left(t, x_{0}\right)\right)=x\left(t, x_{0}\right)^{T} \operatorname{Px}\left(t, x_{0}\right),
$$

it can be easily seen that

$$
\begin{aligned}
V\left(x\left(t, c x_{0}\right)\right) & =x\left(t, c x_{0}\right)^{T} \operatorname{Px}\left(t, c x_{0}\right) \\
& =c^{2} x\left(t, x_{0}\right)^{T} \operatorname{Px}\left(t, x_{0}\right) \\
& =c^{2} V\left(x\left(t, x_{0}\right)\right) .
\end{aligned}
$$

These two lemmas permit deriving the following proposition.

Proposition 1: The energy gain factor $\hat{\eta}\left(x_{0}\right)$ defined in (7) is constant along rays through the origin, i.e.

$$
\hat{\eta}\left(x_{0}\right)=\hat{\eta}\left(c x_{0}\right) \quad, \quad c \in \mathbb{R} .
$$

Proof: From (7) and (11), $\hat{\eta}(\cdot)$ can be written as

$$
\hat{\eta}\left(x_{0}\right)=\frac{\min _{t} V\left(x\left(t, x_{0}\right)\right)}{V\left(x_{0}\right)} .
$$

Therefore, it can be seen that

$$
\begin{aligned}
\hat{\eta}\left(c x_{0}\right) & =\frac{\min _{t} V\left(c x\left(t, x_{0}\right)\right)}{V\left(c x_{0}\right)} \\
& =\frac{\min _{t} c^{2} V\left(x\left(t, x_{0}\right)\right)}{c^{2} V\left(x_{0}\right)} \\
& =\hat{\eta}\left(x_{0}\right) .
\end{aligned}
$$

Taking into account Proposition 1, searching for $\eta^{*}$ requires only working with the states belonging to the unit hypersphere rather than the full state space because states

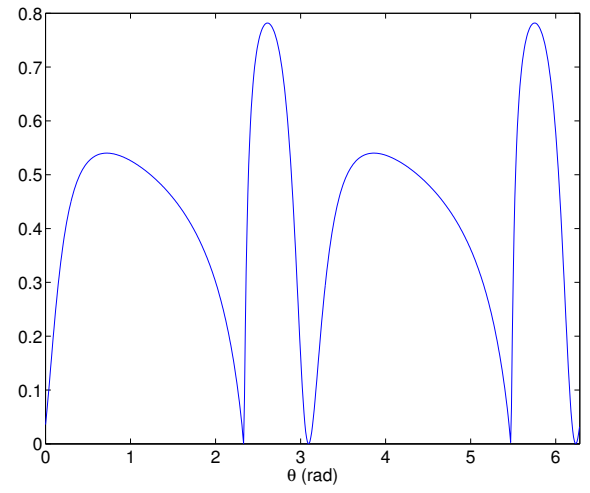

Fig. 6: Values of $\hat{\eta}(\cdot)$ for states lying in the unit circle

lying in the same ray will provide the same value for $\hat{\eta}(\cdot)$. Even more, due to the linear systems symmetry, we only have to explore half hypersphere.

For illustrative purposes, Algorithm 1 shows a simple numerical procedure for finding $\eta^{*}$ for two-dimensional systems, where the only search space is the angle $\theta$. Its extension to higher order systems is straightforward.

Example 6: Following the previous example, Figure 6 illustrates the previous search algorithm in the $[0,2 \pi]$ interval. It plots $\hat{\eta}$ as a function of the state orientation. As it can be seen, the maximum value of $\hat{\eta}$ is $\eta^{*}=0.7818$, and the announced symmetry can be perfectly observed.

\section{APPROXIMATED COMPUTATION OF THE NEXT ACTIVATION TIME}

We are interested in deriving a closed expression that each controller job can use to compute the next job activation time. This will remove the need for the dedicated hardware checking whether the Lyapunov sampling condition holds.

The approach here presented borrows the methodology used in [16] where the approximated computation of the next activation time was addressed for an event-driven condition defined on the error with respect to the sampled state.

Consider that

$$
\dot{x}(t)=f\left(x\left(t, x_{i}\right), u_{i}\right)
$$

is the state variation in the time instant $t$ when the input remains constant. The event condition is

$$
V\left(x\left(t, x_{i}\right)\right)-\eta V\left(x_{i}\right)=0
$$

where for easy explanation, $V(\cdot)$ is restricted to quadratic Lyapunov functions, i.e.

$$
V\left(x\left(t, x_{i}\right)\right)=x\left(t, x_{i}\right)^{T} P x\left(t, x_{i}\right) .
$$

We are interested in finding the minimum $t$ by which (18) holds. Consider the Taylor expansion of $x(\cdot)$

$$
x\left(t, x_{i}\right)=\sum_{n} \frac{1}{n !} \frac{\left.\mathrm{d}^{n} x\left(t, x_{i}\right)\right|_{t_{0}}}{\mathrm{~d} t^{n}}\left(t-t_{0}\right)^{n} .
$$




$$
\begin{gathered}
V\left(x\left(t, x_{i}\right)\right)-\eta V\left(x_{i}\right)=0 \\
x\left(t, x_{i}\right)^{T} P x\left(t, x_{i}\right)-\eta V\left(x_{i}\right)=0 \\
\left(\left.\sum_{n} \frac{1}{n !}(A+B L)^{n} x\left(t, x_{i}\right)\right|_{t_{0}}\left(t-t_{0}\right)^{n}\right)^{T} P\left(\left.\sum_{n} \frac{1}{n !}(A+B L)^{n} x\left(t, x_{i}\right)\right|_{t_{0}}\left(t-t_{0}\right)^{n}\right)-\eta V\left(x_{i}\right)=0 \\
\left(\sum_{n} \frac{1}{n !}\left(\left.x\left(t, x_{i}\right)\right|_{t_{0}}\right)^{T}\left((A+B L)^{n}\right)^{T}\left(t-t_{0}\right)^{n}\right) P\left(\left.\sum_{n} \frac{1}{n !}(A+B L)^{n} x\left(t, x_{i}\right)\right|_{t_{0}}\left(t-t_{0}\right)^{n}\right)-\eta V\left(x_{i}\right)=0 \\
\left.\sum_{n, m} \frac{1}{m ! n !}\left(\left.x\left(t, x_{i}\right)\right|_{t_{0}}\right)^{T}\left((A+B L)^{m}\right)^{T} P(A+B L)^{n} x\left(t, x_{i}\right)\right|_{t_{0}}\left(t-t_{0}\right)^{n+m}-\eta V\left(x_{i}\right)=0
\end{gathered}
$$

For linear systems

$$
\frac{\left.\mathrm{d}^{n} x\left(t, x_{i}\right)\right|_{t_{0}}}{\mathrm{~d} t^{n}}=\left.(A+B L)^{n} x\left(t, x_{i}\right)\right|_{t_{0}}
$$

Recovering (18), we have (22), that in a neighborhood of $t=0$ is

$$
\begin{array}{r}
\sum_{n, m} \frac{1}{m ! n !} x_{i}^{T}\left((A+B L)^{m}\right)^{T} P(A+B L)^{n} x_{i} t^{n+m} \\
-\eta V\left(x_{i}\right)=0 .
\end{array}
$$

Equation (23) is a polynomial of degree $2 n$ for a $n$ order approximation. It can be easily solved for a first order approximation, which yields to a second order equation. In any case, the smallest positive root is the next activation time. Hence, the Lyapunov sampling mechanism permits to adopt a self-triggered scheme for the controller implementation.

The presented method for computing the next activation times, since it uses a Taylor approximation, could provide with times longer than the ones required to retain stability of the system. One possible approach to avoid this problem could be to use a more conservative (bigger) $\eta *$ in the implementation. This would have a double benefit. First, it would decrease inter-sampling times and therefore the likelihood of having longer times than the ones required to retain stability would be eliminated. And second, it would reduce the truncation error. However this has the handicap of increasing the computation demand. Future work will deal with this issue.

\section{CONCLUSIONS AND FUTURE WORK}

This paper has presented a novel sampling approach for event-driven control systems based on Lyapunov functions. The stable Lyapunov sampling mechanism has been introduced. From a theoretical point of view, it ensures infinite samples and therefore, ensures stability for the closed loop trajectory. A technique for finding a self-triggered scheme for the Lyapunov sampling mechanism has also been presented. However, the limitations of the adopted technique require looking for alternative techniques in order to achieve a more accurate and robust self-triggered scheme.

Future work will focus on the computational demand of the introduced sampling mechanism. First, it must be assessed whether the novel sampling scheme is able to reduce the controllers' computational demand compared to the case of periodic controllers, while providing similar performance. Second, free parameters in the Lyapunov sampling scheme, such as the Lyapunov function or the controller can be studied in depth with respect to the resulting controller computational demand.

Finally, future work will also consider the implementation of the Lyapunov sampling mechanism using either dedicated hardware or a suitable self-triggered paradigm.

\section{REFERENCES}

[1] J.A. Stankovic, I. Lee, A. Mok, R. Rajkumar, "Opportunities and obligations for physical computing systems", IEEE Computer Volume 38, Issue 11, pp. 23 - 31, Nov. 2005.

[2] M.S. Branicky, M. Cenk Çavuşoğlu, V. Liberatore "Multi-Disciplinary Challenges and Directions in Networked Cyber-Physical Systems", in NSF Workshop on Cyber-Physical Systems, position paper, 2006

[3] P. Tabuada, "Event-triggered real-time scheduling of stabilizing control tasks," IEEE Trans. on Automatic Control, 52(9), 2007, pp. 1680-1685.

[4] M. Lemmon, T. Chantem, X. S. Hu, and M. Zyskowski, "On selftriggered full information h-infinity controllers," in Proceedings of the $10^{\text {th }}$ International Conference on Hybrib Systems: Computation and Control, Pisa, Italy, Apr. 2007.

[5] W.P.M.H. Heemels, J.H. Sandee, and P.P.J. van den Bosch, "Analysis of event-driven controllers for linear systems," International Journal of Control, 81(4), 2008, pp. 571-590.

[6] V. H. Nguyen and Y. S. Suh, "Networked Estimation with an Areatriggered Transmission Method," Sensors, Vol. 8, No. 2, pp. 897-909, 2008.

[7] T. Henningsson, E. Johannesson, A. Cervin, "Sporadic Event-Based Control of First-Order Linear Stochastic Systems," Automatica, vol. 44, n. 11, pp. 2890-2895, Nov. 2008

[8] Bini, E., Cervin, A., "Delay-aware period assignment in control systems," In Proceedings of the $29^{\text {th }}$ IEEE Real-Time Systems Symposium, Barcelona, Spain (2008)

[9] F. Zhang, K. Szwaykowska, W. Wolf, and V. Mooney, "Task Scheduling for Control Oriented Requirements for Cyber-Physical Systems," in 29th IEEE Real-Time System Symposium, Barcelona, Spain, Dec. 2008.

[10] M.Rabi, K.H. Johansson, M. Johansson, M., "Optimal stopping for event-triggered sensing and actuation," 47th IEEE Conference on Decision and Control, 2008.

[11] P. Martí, C. Lin, S. Brandt, M. Velasco, and J.M. Fuertes, "Draco: Efficient Resource Management for Resource-Constrained Control Tasks," IEEE Transactions on Computers, Jan. 2009.

[12] S. Zhuang, and C.G. Cassandras, "Optimal Control of Discrete Event Systems with Weakly Hard Real-Time Constraints," Discrete Event Dynamic Systems, 19, 1, 67-89, Mar. 2009.

[13] M-M. Ben Gaid, A. Çela, and Y. Hamam, "Optimal Real-Time Scheduling of Control Tasks with State Feedback Resource Allocation," IEEE Transactions on Control Systems Technology, Vol. 17, n. 2, pp. 309-326, Mar. 2009.

[14] X. Wang and M. Lemmon, Self-triggered Feedback Control Systems with Finite-Gain L2 Stability, IEEE Transactions on Automatic Control, vol. 45, n. 3, pp. 452-467, March 2009.

[15] X. Wang and M. Lemmon, "Event Design in Event-Triggered Feedback Control Systems," IEEE Conference on Decision and Control, December 2008.

[16] M. Velasco, P. Martí and E. Bini, "Control-driven Tasks: Modeling and Analysis", in 29th IEEE Real-Time Systems Symposium, 2008

[17] M. Velasco, P. Martí and J.M. Fuertes, "The Self Triggered Task Model for Real-Time Control Systems," in WiP of the 24th IEEE Real-Time Systems Symposium, December 2003.

[18] A. Anta and P. Tabuada, "Space-time scaling laws for self-triggered control," in Proceedings of the 47th IEEE Conference on Decision and Control, December 2008. 